Article

\title{
Positive Response to Thermobalancing Therapy Enabled by Therapeutic Device in Men with Non-Malignant Prostate Diseases: BPH and Chronic Prostatitis
}

\author{
Ivan Gerasimovich Aghajanyan ${ }^{1}$ and Simon Allen ${ }^{2, *}$ \\ 1 Department of Urology, Yerevan State Medical University, 9 Ezras Hasratyan St, Yerevan 0052, Armenia; \\ aghajanyan_hov@yahoo.com \\ 2 Fine Treatment, 29 Rewley Road, Oxford OX1 2RA, UK \\ * Correspondence: info@finetreatment.com; Tel.: +44-795-887-8300; Fax: +44-186-572-8255
}

Academic Editor: Maurizio Battino

Received: 22 February 2016; Accepted: 14 April 2016; Published: 18 April 2016

\begin{abstract}
Background: The most common types of non-malignant prostate diseases are benign prostatic hyperplasia (BPH) and chronic prostatitis $(\mathrm{CP})$. The aim of this study was to find out whether thermobalancing therapy with a physiotherapeutic device is effective for BPH and CP. Methods: During a 2.5-year period, 124 men with BPH over the age of 55 were investigated. Clinical parameters were tested twice: via the International Prostate Symptom Score (IPSS) and via ultrasound measurement of prostate volume (PV) and uroflowmetry maximum flow rate $\left(\mathrm{Q}_{\mathrm{max}}\right)$, before and after six months of therapy. In 45 men with CP under the age of 55, the dynamics of the National Institute of Health Chronic Prostatitis Symptom Index (NIH-CPSI) were studied. Results: The results of the investigated index tests in men with BPH confirmed a decrease in IPSS $(p<0.001)$, a reduction in PV $(p<0.001)$, an increase in $\mathrm{Q}_{\max }(p<0.001)$, and an improvement of quality of life (QoL) $(p<0.001)$. NIH-CPSI scores in men with $\mathrm{CP}$ indicated positive dynamics. Conclusions: The observed positive changes in IPSS, PV, and $\mathrm{Q}_{\max }$ in men with BPH and the improvement in NIH-CPSI-QoL in patients with $\mathrm{CP}$ after using a physiotherapeutic device for six months as mono-therapy, support the view that thermobalancing therapy with the device can be recommended for these patients. Furthermore, the therapeutic device is free of side effects.
\end{abstract}

Keywords: chronic prostatitis; CP/CPPS; chronic pelvic pain; benign prostatic hyperplasia; $\mathrm{BPH}$; prostate natural treatment; therapeutic device; thermobalancing therapy; prostatic disease

\section{Introduction}

The prevalence of histologically diagnosed prostatic hyperplasia increases from 8 percent in men aged 31-40, to 40-50 percent in men aged 51-60, and to over 80 percent in men older than age 80 [1]. Epidemiologic data suggest that the prevalence of chronic prostatitis $(\mathrm{CP})$ is comparable to ischemic heart disease and diabetes mellitus, as about $8.2 \%$ men are believed to be affected [2]. Thus, benign prostate hyperplasia (BPH) and $\mathrm{CP}$ are common diseases in males, and $\mathrm{BPH}$ is often complicated by $\mathrm{CP}$ [3]. At the same time, chronic inflammation in the prostate gland plays an important role in the development of BPH [4]. Moreover, inflammation may be considered a key component of BPH pathogenesis, in addition to androgen receptor signaling in tissue remodeling typical of the advanced stages of the disease [5].

Accumulating evidence suggests that inflammation may contribute to the development of BPH and LUTS. The inflammatory injury may contribute to cytokine production via inflammatory cells driving local growth factor production and angiogenesis in the prostatic tissue. Acceptance of this 
suggestion can lead to novel treatment strategies [6]. The first-line treatments in $\mathrm{CP}$ have always been antibiotics and non-steroidal anti-inflammatory drugs (NSAIDs) [7]. A new potential target for medical therapy of LUTS due to BPH is $\mathrm{CP}$ and consequently prostatic inflammation. Drugs currently investigated for the treatment of this inflammation include the hexanic lipidosterolic extract of Serenoa repens, nonsteroidal anti-inflammatory drugs, and vitamin D receptor agonists [8].

Although there are some suggestions that NSAIDs increase the risk of prostatic diseases, most studies suggest that NSAIDs have the potential to improve symptoms in, and reduce the risk of, prostatic diseases [9]. The systematic review has shown that NSAIDs compared to a placebo, or in addition to known BPH therapy, improve BPH symptoms as measured using the IPSS and peak urinary flow rate, so authors believe that their study provides proof of the concept that inflammation in BPH can be a real target for medical research and targeted intervention [10].

However, non-selective NSAIDs should be used with caution, especially in older people, after other safer treatments have not provided sufficient pain relief. The lowest dose should be provided, and for the shortest duration. NSAIDs should be prescribed especially carefully to older people, with monitoring for gastrointestinal, renal and cardiovascular side effects, and drug-disease interactions [11].

At the same time, since 2009, when Simon Allen invented the non-invasive and safe thermobalancing therapy, enabled by the use of a therapeutic device for the treatment of chronic internal diseases, empirical data were obtained that the device he designed provides the positive and clinically meaningful effect in men with $\mathrm{CP}$ and benign prostatic hyperplasia [12]. A clinically controlled trial involving 124 men with $\mathrm{BPH}$, to whom the therapeutic device was administered exclusively as a mono-therapy, has confirmed the effectiveness and safety of thermobalancing therapy [13]. A clinical study on 45 men with CP has also been completed. Thus, in the present study, we investigated the effect of the continuous use of the therapeutic device on patients with prostatic disease.

\section{Materials and Methods}

\subsection{Study Design}

The observational clinical controlled study was used. Enrollment began on April 2013 in the Department of Urology of the Yerevan State Medical University. The Ethics Committee of the Yerevan State Medical University approved the clinical study on thermobalancing therapy enabled by the use of a physiotherapeutic device. Effectiveness of the therapeutic device was studied by comparing men with $\mathrm{BPH}$, who received treatment with the therapeutic device, with a control group in the watchful waiting stage, and by also comparing patients with $\mathrm{CP}$ who received treatment with the therapeutic device with the control no-treatment group. Dynamics of the symptoms and the indicators in each group were evaluated in comparison to their data in the beginning and end of the treatment, before and after a 6-month period of time.

\subsection{Evaluation}

The baseline evaluations included a complete physical examination, medical history, DRE, serum biochemistry, PSA measurements, electrolytes, and urine and renal function tests. Evaluations were made at baseline and 6 months after the treatment. International Prostate Symptom Score-Quality of Life (IPSS-QoL) scores were used in men with BPH, and the National Institute of Health Chronic Prostatitis Symptom Index (NIH-CPSI) scores were used in patients with CP. Prostate volume (PV) was measured at baseline and at 6 months after the treatment via ultrasonography (US-9000E2 ultrasound scanner, Rising Medical Equipment Co. Ltd., Beijing, China), and uroflowmetry (maximum urinary flow rate- $\mathrm{Q}_{\max }, \mathrm{mL} / \mathrm{s}$ ) was used for the measurement of the rate of urine flow parameters (Sanuro2UL, Santron Meditronic, Maharashtra, India). The standard ellipsoid formula length $\times$ width $\times$ height $\times 0.52$ was used to determine prostate volume. 


\subsection{Participants and Interventions}

226 men were examined in April 2013, and 124 with BPH were selected for the clinical trial. Inclusion criteria: Men were eligible for enrolment if they were over the age of 55, in the absence of acute prostatitis, at the level of prostate-specific antigen (PSA) not more than $4 \mathrm{~m} \cdot \mathrm{mol} / \mathrm{L}$, and with a Qmax higher than $5 \mathrm{~mL} / \mathrm{s}$. It did not matter if they were treated at the time of enrollment with medicines. However, after the use of the therapeutic device, other treatments were cancelled gradually. Exclusion criteria: PV greater than $60 \mathrm{~mL}$ and co-morbidities, such as diabetes, heart failure, cancer, etc.

Thus, 80 men were excluded, as their PV was over $60 \mathrm{~mL}$ or they had severe co-morbidities; 10 preferred an operation; four were suspected of prostate cancer; and eight did not attend the following examinations. The quantity of patients in the treatment and control groups was similar (124 men).

In this study, we also present part of another study that included the dynamic of quality of life (QoL) in 45 patients with chronic nonbacterial prostatitis (Cat III), also known as CP/CPPS, before and after the 6-month period of the use of the physiotherapeutic device, and in 45 men in the control group that did not use the device. Men in treatment groups were given therapeutic device, termed Allen's Device (see Figure 1).

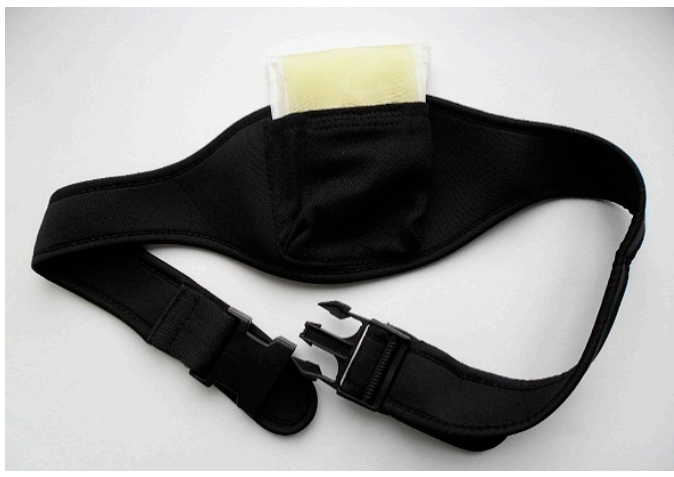

(A)

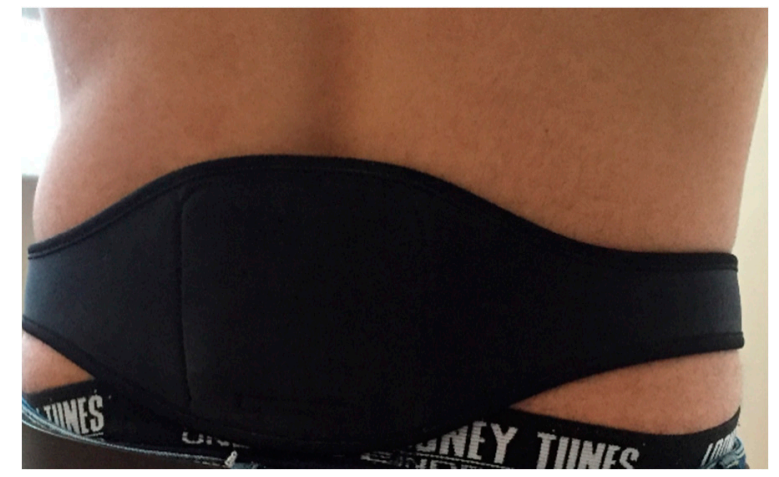

(B)

Figure 1. The therapeutic device alone (on the $\mathbf{A}$ ) and the device tightly applied to the coccyx area in the projection of the prostate (on the $\mathbf{B}$ ).

\subsection{Statistical Analysis}

Because the independent samples $t$-test and pair samples $t$-test is only suitable for interval and ratio data, the Wilcoxon signed-rank test by using SPSS was conducted.

\section{Results}

\subsection{Urinary Symptoms and QoL}

In the control group, the mean IPSS-UrS increased from $13.45 \pm 3.254$ to $14.35 \pm 3.396$, whereas in the treatment group the mean IPSS-UrS decreased from $14.33 \pm 3.399$ to $4.73 \pm 2.754$ at the end of the observation period. For the control group, the $\mathrm{z}$ value was 6.018 with a $p$ value $(p<0.001)$. For the treatment group, the $\mathrm{z}$ value was 9.674 with a significance level $(p<0.001)$. This indicates that the treatment with the therapeutic device decreased the urinary symptoms significantly, while in the absence of treatment the symptoms increased significantly, see Figure 2.

In the control group, the mean IPSS-QoL increased from $3.43 \pm 0.956$ to $3.76 \pm 0.983$, whereas in the treatment group the mean IPSS-QoL decreased from $3.91 \pm 0.755$ to $1.39 \pm 1.110$. For the control group, the $\mathrm{z}$ value was 5.286 with a $p$ value $(p<0.001)$. For the treatment group, the $\mathrm{z}$ value was 9.672 with a $p$ value $(p<0.001)$. These results indicate that the treatment with the therapeutic device improved the QoL, while in the control group the QoL worsened, see Figure 2. 


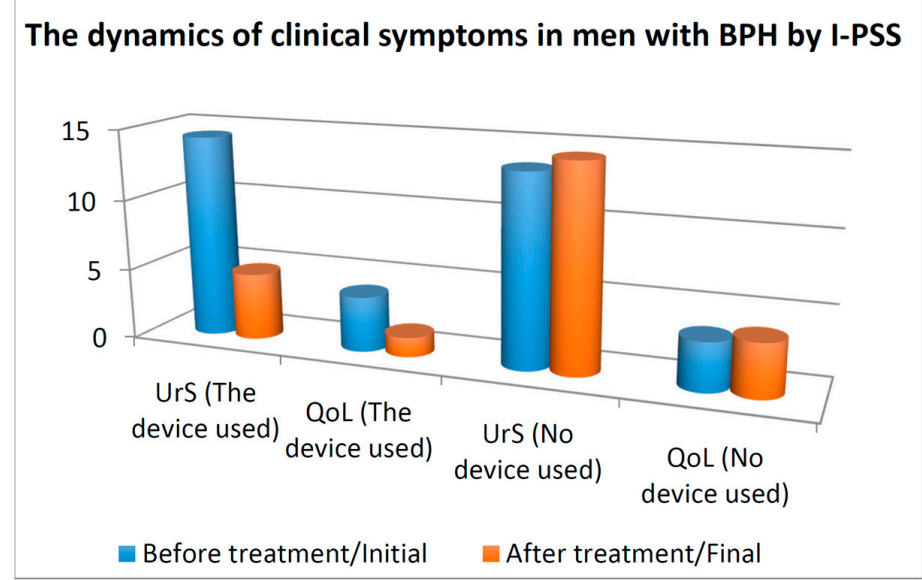

Figure 2. International Prostate Symptom Score (IPSS), urinary symptoms (UrS), and quality of life (QoL) in 124 patients with benign prostate hyperplasia (BPH) on thermobalancing therapy and in the control group, 124 men with BPH in watchful waiting at the beginning and at the end of the study.

\subsection{Prostate Volume and $Q_{\max }$}

In the control group the mean prostate volume increased from 45.54 to $50.85 \mathrm{~mL}$, whereas in the treatment group the mean prostate volume decreased from 45.19 to $31.86 \mathrm{~mL}$. For the control group, the $\mathrm{z}$ value is -8.727 at the significance level $(p<0.001)$. Thus, there was a statistically significant increase in the prostate volume in the control group. For the treatment group, the $z$ value is -9.669 at the significance level $(p<0.001)$. Thus, the treatment with the therapeutic device reduced the PV level significantly, whereas in the no-treatment group the PV level increased.

In the control group, the mean $Q_{\max }$ decreased from $7.95 \pm 2.871$ to $7.7 \pm 2.695 \mathrm{~mL} / \mathrm{s}$, whereas in the treatment group the mean $Q_{\max }$ increased from $8.10 \pm 3.041$ to $17.73 \pm 4.392 \mathrm{~mL} / \mathrm{s}$. For the control group, the $z$ value was 1.929 and the $p$ value was $0.054(>0.05)$, indicating no statistically significant difference. For the treatment group, the $\mathrm{z}$ value is 9.621 at the significance level $(p<0.001)$, indicating a significant increase in the $\mathrm{Q}_{\max }$. Thus, our results demonstrate that the therapeutic device increased the uroflowmetry $Q_{\max }$ significantly in BHP patients, whereas the control group had no significant difference in the uroflowmetry $Q_{\max }$. See changes in $P V$ and $Q_{\max }$ on Figure 3.

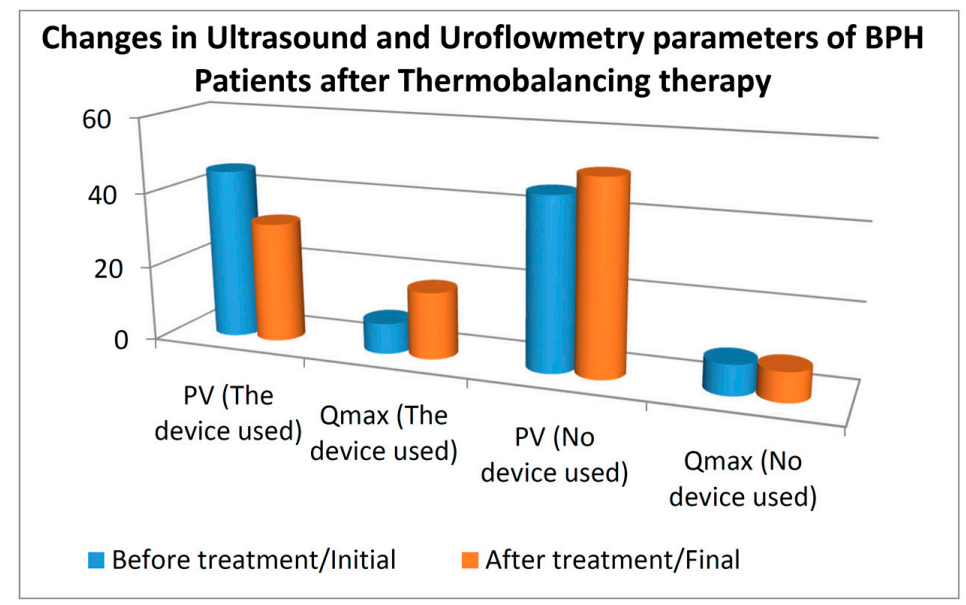

Figure 3. The changes in prostate volume $(\mathrm{PV}) \mathrm{mL}$ and the uroflowmetry (maximum urinary flow rate $\left(\mathrm{Q}_{\max }, \mathrm{mL} / \mathrm{s}\right)$ in 124 patients with $\mathrm{BPH}$ on thermobalancing therapy and in the control group, 124 men with $\mathrm{BPH}$ in watchful waiting, at the beginning and at the end of the study. 


\subsection{Quality of Life Score in Patients with Chronic Prostatitis}

We assessed the QoL according to NIH-CPSI (Figure 4). In the control group, the mean QoL decreased slightly from 8.47 to 8.33 , whereas in the treatment group the mean QoL decreased from 8.11 to 2.98 . For the control group, the $\mathrm{z}$ value was -0.420 at the significance level of 0.675 with a $p$ value $>0.05$. For the treatment group, the $\mathrm{z}$ value was -5.661 at the significance level $p$ value $<0.001$. These results indicate that the treatment with the therapeutic device decreased the QoL score significantly, while in the control group it decreased slightly, see Figure 4.

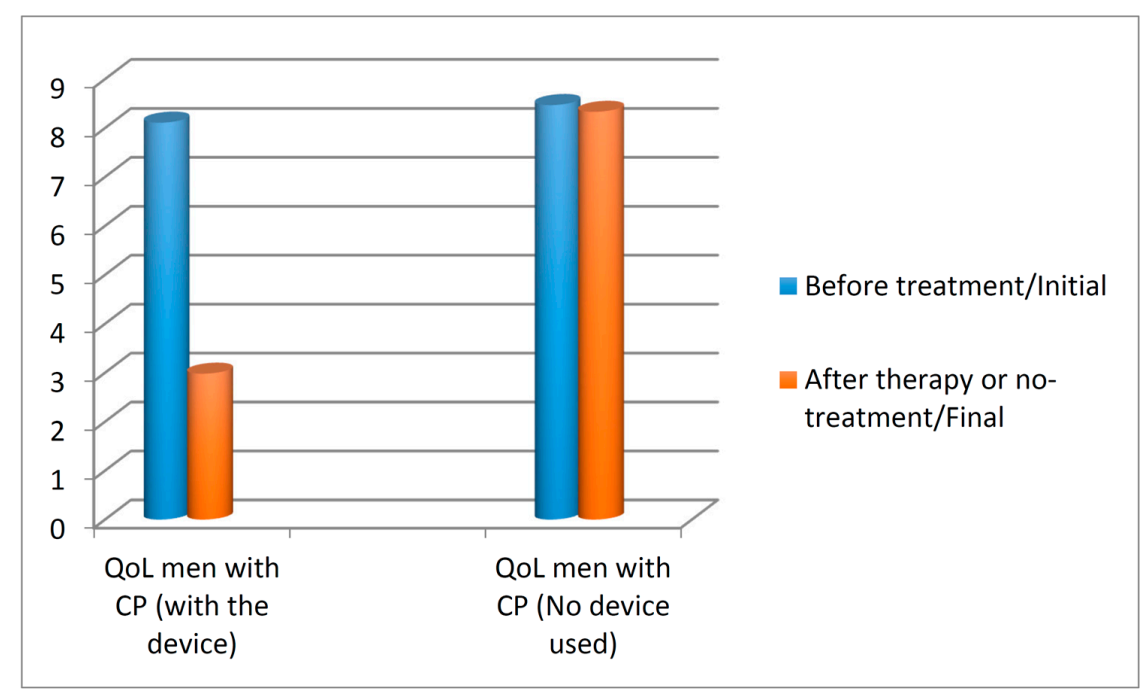

Figure 4. National Institute of Health Chronic Prostatitis Symptom Index (NIH-CPSI)—Quality of Life (QoL) score in 45 patients with chronic prostatitis/chronic pelvic pain syndrome (CP/CPPS) on thermobalancing therapy and in the control group, 45 men with CP/CPPS with no treatment, at the beginning and at the end of the study.

\section{Discussion}

Examined before and after a 6-month treatment period with the therapeutic device, the patients with BPH reported a significant improvement to the disturbing LUTS and QoL. Moreover, after the use of the therapeutic device the PV level decreased and uroflowmetry $Q_{\max }$ significantly increased. Changes in NIH-CPSI-QoL scores have shown the positive results in improving QoL. More details on the influence of thermobalancing therapy on men with $\mathrm{CP}$ will be discussed in another article. At the same time, in the no-treatment groups the symptoms and parameters worsened.

These obtained data indicate that thermobalancing therapy is effective for BPH and CP. The observational clinically controlled study was used on both separate trials for CP and BPH. The studies were not randomized, which may limit the accuracy of the results. Of course, having "placebo" or "sham" group as controls could provide more confidence in the outcomes. However, most men with $\mathrm{CP}$ and pelvic pain have serious mental problems, so some experts believe that psychological problems are part of the cause of CP/CPPS [14]. Therefore, it was difficult to suggest to men with CP/CPPS that they wear something useless for six months. Usually, patients with CP/CPPS felt healthier within weeks; therefore, they used the device as was required. We had similar thoughts about patients with an enlarged prostate, as the health-related QoL of patients with BPH is considered poor, and their psychological well-being severely affected. Postvoid residual urine, lower urinary tract symptoms, anxiety, and depression are identified as being significant predictive factors of the health-related QoL of patients with an enlarged prostate [15].

LUTS associated with BPH is a highly impactful condition that is often undertreated. LUTS/BPH have a major impact on men, their families, health services, and society. Men with LUTS secondary to $\mathrm{BPH}$ should not simply accept their symptoms as part of the ageing process, but should be encouraged 
to consult their physicians if they have bothersome symptoms [16]. In the last decade, the opinion of the necessity of medical/surgical treatment of BPH has been challenged. BPH/LUTS should not be viewed as an inevitable disease of older people but as part of the aging process which can be prevented [17]. Moreover, research that has included a total of 2,620,269 patients with BPH who were treated within 5 years has shown that medical treatment was interrupted for approximately $16 \%$ of patients. Therefore, it is necessary to improve the level of care for men with BPH [18].

As thermobalancing therapy is free from side effects, it can play an important role in the prevention of BPH development and progression. Thermobalancing therapy is entirely different from common heating treatments because it regulates the affected organ's temperature locally, maintaining it within the normal body temperature range. All other thermotherapies can damage organs, because the high temperatures injure normal cells. On the other hand, low temperatures decrease cellular metabolism and, as a result, interfere with natural replenishment and healing.

The therapeutic device tightly applies the thermoelement to the skin in the projection of the prostate overcoming the skin barrier spreading energy inside the body. Allen hypothesized that this therapy long-term effect on the prostate improves blood circulation at the capillary level. This view is supported by various data; for instance, in the last decade, the pathogenesis of BPH began to be considered from the perspective of vascular dysfunction [19], chronic ischemic tissue [20], and increasing the pressure in the prostate [21].

However, other investigators state that, apart from the old concept of mainly hormone-dependent prostate growth, new ideas about the cause of BPH include an inflammatory factor [22]. Therefore, materials presented at a satellite symposium entitled, "Inflammation and Prostatic Diseases: From Bench to Bedside," held during the 2015 annual meeting of the European Association of Urology in Madrid, Spain, revealed that reducing chronic inflammation is a target for the treatment of BPH [23].

NSAIDs are commonly used as anti-inflammatory drugs worldwide, but these medications are associated with side effects in the gastrointestinal (GI) tract. Furthermore, these drugs can damage gastric and duodenal mucosa, and even the esophagus [24]. Certain NSAIDs can increase the risk of heart attack and stroke, especially in long-term use; therefore, prescription of this medication should be avoided in patients at high risk of cardiovascular disease [25].

The therapeutic device, in contrast with NSAIDs and long-term BPH medications, provides side-effect-free treatment that targets the pathological nidus in the prostate continuously for a prolonged period of time, i.e., for days, months, or even years, maintaining the accumulated temperature. Allen's research on the origin of diseases suggests the causal root of chronic internal diseases, including prostate enlargement, namely capillary expansion.

This conclusion is based on two functional physiological properties of capillaries which are activated by an irritating factor, i.e., a trigger. The constriction of capillaries in response to an irritating trigger develops local micro-hypothermia. It is this focus on hypothermia, which in turn becomes a constant irritant maintaining illness, making a disease chronic. In response to irritation (i.e., a trigger-initiator and later focus on hypothermia) and in order to eliminate them, the blood flow increases through the spontaneous expansion of the capillary net locally. The formation of new capillaries is essentially the growth of the excess tissue that leads to an increased pressure inside the prostate and its enlargement.

For the purpose of a targeted treatment for the secondary focus on hypothermia inside the organ, Allen suggested thermobalancing therapy enabled by a therapeutic device. This device contains a natural thermoelement which accumulates body heat, becoming a source of energy itself. There is no battery or electricity involved. Moreover, it should be noted that treatments with imposed heat over $40{ }^{\circ} \mathrm{C}$ (104 Fahrenheit) can be damaging, because the high temperatures destroy delicate cells of the living organism. We believe that the use of the therapeutic device by keeping the right temperature in the projection of the prostate gland improves blood circulation at the capillary level, stopping and reversing prostate swelling and enlargement, and easing the $\mathrm{BPH}$ and $\mathrm{CP}$ symptoms. 


\section{Conclusions}

Both clinical trials of CP and BPH highlight that the therapeutic device is potentially able to target pathogenetic component in the prostate gland. Thermobalancing therapy is a new prospect for safe and effective physiotherapeutic intervention in BPH treatment. The reduction of the PV after use of the therapeutic device, termed Allen's Device, correlates with a significant decrease in urinary symptoms and an improvement of QoL in patients with an enlarged prostate and CP.

Author Contributions: Both authors made substantive intellectual contributions to the presented study. They have made substantial contributions to the conception and design and to the acquisition and interpretation of data, have been involved in drafting the manuscript and revising it critically for important intellectual content, and have given final approval of the version to be published.

Conflicts of Interest: The authors declare that they have no competing interests. Simon Allen applied to USPTO in 2009 - “Therapeutic Device and Method"—but has not received reimbursements, fees, funding, or salary relating to the content of the manuscript.

\section{References}

1. Epidemiology and Pathogenesis of Benign Prostatic Hyperplasia. Available online: http://www.upto date.com/contents/epidemiology-and-pathogenesis-of-benign-prostatic-hyperplasia (accessed on 11 August 2015).

2. Magistro, G.; Wagenlehner, F.M.; Grabe, M.; Weidner, W.; Stief, C.G.; Nickel, J.C. Contemporary management of chronic prostatitis/chronic pelvic pain syndrome. Eur. Urol. 2016, 69, 286-297. [CrossRef] [PubMed]

3. Ma, Y.; Zhang, W.L. Benign prostate hyperplasia with chronic prostatitis: An update. Zhonghua Nan Ke Xue 2010, 16, 646-650. [PubMed]

4. Shoskes, D.A.; Prots, D.; Karns, J.; Horhn, J.; Shoskes, A.C. Greater endothelial dysfunction and arterial stiffness in men with chronic prostatitis/chronic pelvic pain syndrome-A possible link to cardiovascular disease. J. Urol. 2011, 186, 907-910. [CrossRef] [PubMed]

5. Chughtai, B.; Lee, D.; Te, A.; Kaplan, S. Role of inflammation in benign prostatic hyperplasia. Rev. Urol. 2011, 13, 147-150. [PubMed]

6. Bostanci, Y.; Kazzazi, A.; Momtahen, S.; Laze, J.; Djavan, B. Correlation between benign prostatic hyperplasia and inflammation. Curr. Opin. Urol. 2013, 23, 5-10. [CrossRef] [PubMed]

7. Stevermer, J.J.; Easley, S.K. Treatment of prostatitis. Am. Fam. Physician 2000, 61, 3015-3022. [PubMed]

8. Ficarra, V.; Rossanese, M.; Zazzara, M.; Giannarini, G.; Abbinante, M.; Bartoletti, R.; Mirone, V.; Scaglione, F. The role of inflammation in lower urinary tract symptoms (LUTS) due to benign prostatic hyperplasia (BPH) and its potential impact on medical therapy. Curr. Urol. Rep. 2014, 15, 463. [CrossRef] [PubMed]

9. Ishiguro, H.; Kawahara, T. Nonsteroidal anti-inflammatory drugs and prostatic diseases. BioMed Res. Int. 2014. [CrossRef] [PubMed]

10. Kahokehr, A.; Vather, R.; Nixon, A.; Hill, A.G. Non-steroidal anti-inflammatory drugs for lower urinary tract symptoms in benign prostatic hyperplasia: Systematic review and meta-analysis of randomized controlled trials. BJU Int. 2013, 111, 304-311. [CrossRef] [PubMed]

11. Abdulla, A.; Adams, N.; Bone, M.; Elliott, A.M.; Gaffin, J.; Jones, D.; Knaggs, R.; Martin, D.; Sampson, L.; Schofield, P. Guidance on the management of pain in older people. Age Ageing 2013, 42, i1-i57. [CrossRef] [PubMed]

12. Allen, S.; Adjani, A. Therapeutic Device and Method. US 20110152986 A1, 2009.

13. Allen, S.; Aghajanyan, I.G. Benign prostatic hyperplasia treatment with new physiotherapeutic device. Urol. J. 2015, 12, 2371-2376. [PubMed]

14. Chung, S.D.; Lin, H.C. Association between chronic prostatitis/chronic pelvic pain syndrome and anxiety disorder: A population-based study. PLoS ONE 2013, 8, e64630. [CrossRef] [PubMed]

15. Pinto, J.D.; He, H.G.; Chan, S.W.; Wang, W. Health-related quality of life and psychological well-being in men with benign prostatic hyperplasia: An integrative review. Jpn. J. Nurs. Sci. 2016. [CrossRef] [PubMed]

16. Speakman, M.; Kirby, R.; Doyle, S.; Ioannou, C. Burden of male lower urinary tract symptoms (LUTS) suggestive of benign prostatic hyperplasia (BPH)—Focus on the UK. BJU Int. 2015, 115, 508-519. [CrossRef] [PubMed] 
17. Corona, G.; Vignozzi, L.; Rastrelli, G.; Lotti, F.; Cipriani, S.; Maggi, M. Benign prostatic hyperplasia: A new metabolic disease of the aging male and its correlation with sexual dysfunctions. Int. J. Endocrinol. 2014, 2014, 329456. [CrossRef] [PubMed]

18. Lukacs, B.; Cornu, J.N.; Aout, M.; Tessier, N.; Hodée, C.; Haab, F.; Cussenot, O.; Merlière, Y.; Moysan, V.; Vicaut, E. Management of lower urinary tract symptoms related to benign prostatic hyperplasia in real-life practice in France: A comprehensive population study. Eur. Urol. 2013, 64, 493-501. [CrossRef] [PubMed]

19. Shimizu, S.; Tsounapi, P.; Shimizu, T.; Honda, M.; Inoue, K.; Dimitriadis, F.; Saito, M. Lower urinary tract symptoms, benign prostatic hyperplasia/benign prostatic enlargement and erectile dysfunction: Are these conditions related to vascular dysfunction? Int. J. Urol. 2014, 21, 856-864. [CrossRef] [PubMed]

20. Andersson, K.; Nomiya, M.; Yamaguchi, O. Chronic pelvic ischemia: Contribution to the pathogenesis of lower urinary tract symptoms (LUTS): A new target for pharmacological treatment? LUTS 2014. [CrossRef] [PubMed]

21. Cohen, P.G. Abdominal obesity and intra-abdominal pressure: A new paradigm for the pathogenesis of the hypogonadal-obesity-BPH-LUTS connection. Horm. Mol. Biol. Clin. Investig. 2012, 11, 317-320. [CrossRef] [PubMed]

22. Füllhase, C.; Hakenberg, O. New concepts for the treatment of male lower urinary tract symptoms. Curr. Opin. Urol. 2015, 25, 19-26. [CrossRef] [PubMed]

23. Jack, A.S. Inflammation in the pathophysiology of benign prostatic hypertrophy. Eur. Urol. Suppl. 2015, 14, 1455-1458.

24. Sostres, C.; Gargallo, C.J.; Lanas, A. Nonsteroidal anti-inflammatory drugs and upper and lower gastrointestinal mucosal damage. Arthritis Res. Ther. 2013, 15. [CrossRef] [PubMed]

25. McGettigan, P.; Henry, D. Use of non-steroidal anti-inflammatory drugs that elevate cardiovascular risk: An examination of sales and essential medicines lists in low-, middle-, and high-income countries. PLoS Med. 2013, 10, e1001388. [CrossRef] [PubMed]

(C) 2016 by the authors; licensee MDPI, Basel, Switzerland. This article is an open access article distributed under the terms and conditions of the Creative Commons Attribution (CC-BY) license (http://creativecommons.org/licenses/by/4.0/). 